\title{
EVOLUCIÓN GEOMORFOLÓGICA DEL CONJUNTO VOLCÁNICO SUBHISTÓRICO DE MONTAÑA CASCAJO (DORSAL DE BILMA-ABEQUE) EN TENERIFE
}

\author{
Fco. Javier Dóniz Páez \\ José Enrique Coello de la Plaza \\ Geógrafos \\ Universidad de La Laguna
}

\section{RESUMEN}

Con cierta frecuencia, tanto en geografía como en otras disciplinas científicas, se hace alusión a determinados paisajes y lugares como verdaderos laboratorios para la investigación. El conjunto volcánico de Montaña Cascajo, dada la profusión de elementos y formas estructurales y de modelado, constituye, sino el mejor, uno de los espacios más interesantes, junto con los volcanes históricos, de la geografía insular para establecer la evolución geomorfológica en corteza de reciente creación y determinar como se transforman los rasgos morfológicos originales, qué tipo de factores son responsables de esa dinámica, cuáles son los procesos que introducen variaciones significativas en la fisonomía del conjunto eruptivo y que formas generan. La descripción detallada de sus rasgos morfológicos permite, sin duda, establecer aproximaciones a un modelo de evolución geomorfológica de los volcanes subhistóricos que pueda ser más o menos generalizado a otros volcanes simples de edades muy recientes, holocenos, y afectados por condiciones medio-ambientales propias de piso montano seco de Tenerife.

Palabras clave: Geomorfología volcánica, volcanismo reciente, evolución geomorfológica, volcán escoriáceo, Montaña Cascajo, Tenerife, Canarias.

\section{ABSTRACT}

Certain types of landscapes frecuently serve as empirical laboratories both in geography and in other sciences. Given its shape and distribution of structures, the Montaña Cascajo volcanic complex in Tenerife, with the historical volcanoes, constitutes one of the most interesting localities for studying the geomorphological evolution of a recently formed oceanic island. This report determines the changes of the original morphological features, evaluating the factors and processes that are causing important variations in the 
appearance of the eruptive complex, and the cone shapes that are generated. The detailed description of the morphology of Montaña Cascajo allows the adoption of an evolutionary model of a prehistoric volcano. In a generalized way, this model also explains the geomorphological evolution of other recent and Holocene volcanic cones in Tenerife that have been exposed to arid mountain conditions.

Key words: Volcanic geomorphology, recent volcanism, geomorphological evolution, scoria cone, Cascajo Mountain, Tenerife. Canary Islands.

\section{Introducción}

Las investigaciones sobre la evolución de las formas del relieve se han convertido en uno de los temas centrales de los análisis geomorfológicos. Los paisajes volcánicos de reciente creación ofrecen una buena oportunidad para estudiar la dialéctica que se establece entre los rasgos morfológicos originales y los procesos iniciales de desmantelamiento posteruptivos, a la vez que permiten evaluar tanto las transformaciones fisonómicas de los volcanes como su dinámica geomorfológica.

La erupción de Montaña Cascajo tuvo lugar en torno al año 300 d.n.e. (Soler y Carracedo; 1986) en el eje de la morfoestructura eruptiva de Bilma-Abeque y se generó a
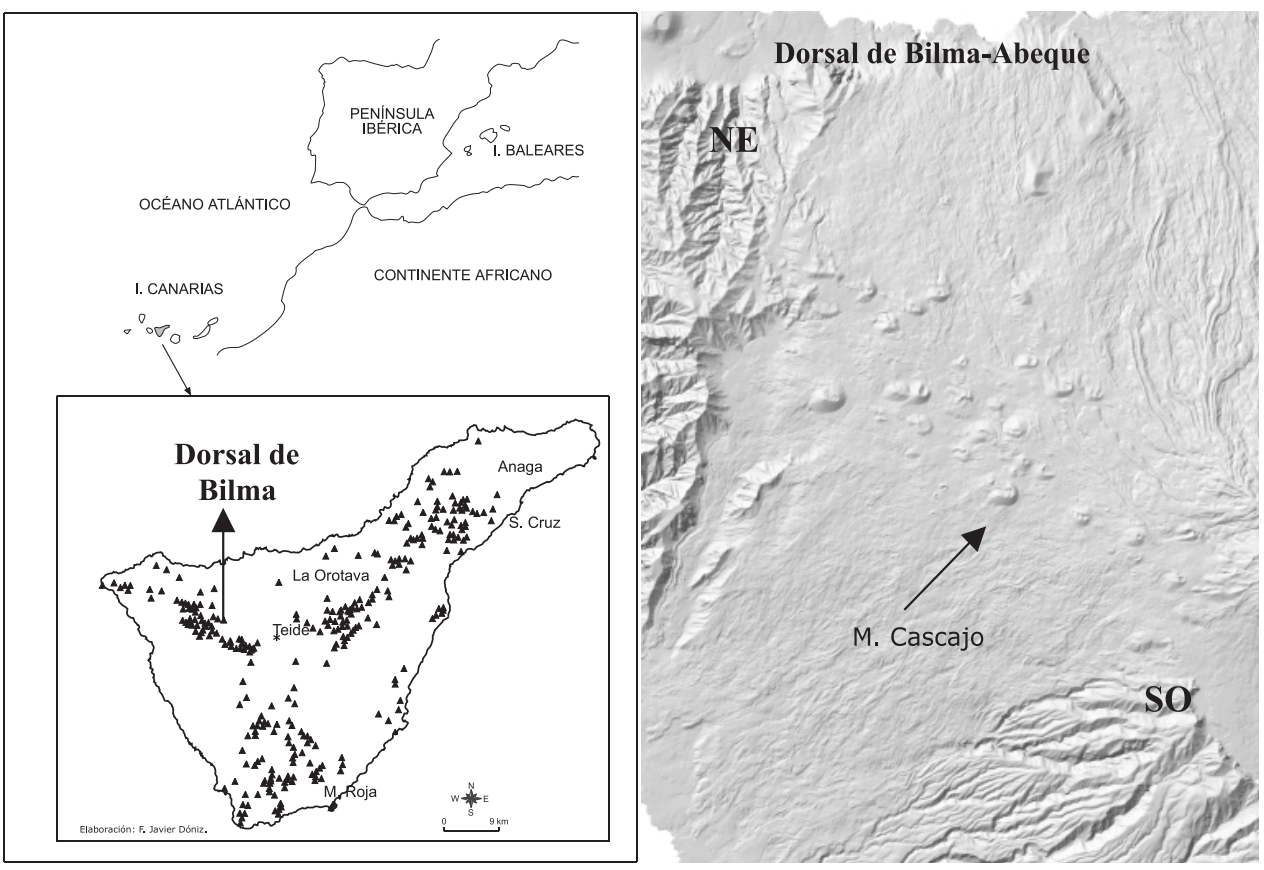

Figura 1. Localización de la isla de Tenerife y de la dorsal de Bilma con la distribución espacial de los volcanes basálticos mogénicos recientes (izquierda). Modelo digital del terreno de la dorsal de Bilma-Abeque con la ubicación de M. Cascajo (derecha) 
partir de fracturas lineales fundamentalmente de rumbo noreste-suroeste. Esta dorsal volcánica dispone de una superficie aproximada de unos $86,94 \mathrm{~km}^{2}$ y cuenta con el mayor número de volcanes basálticos simples de edades muy recientes de Tenerife (46 edificios) (Dóniz, 2004), acogiendo a cuatro de los siete subhistóricos ${ }^{1}$ (Samara, Cascajo, Boca Cangrejo y Montañas Negras) y dos de los seis (Garachico y Chinyero) fechados después de la anexión de las islas a la Corona de Castilla, realizada entre 1402 y 1493.

La dorsal de Bilma-Abeque corresponde a una edificación compleja, de génesis volcánica, que se construye a partir de numerosos episodios monogénicos, siguiendo una única línea tectónica de marcado carácter rectilíneo de rumbo NE-SO, aunque dada su juventud geológica, la concentración de los procesos eruptivos propicia la formación de un edificio en tejado a dos aguas poco resaltado (Dóniz, 2004). Geomorfológicamente, muestra como es característico de las dorsales, dos ámbitos bien diferenciados: una línea de cumbres que constituye el eje de la estructura y en la cual se concentra la mayor parte de los aparatos volcánicos, y un dorso constituido en lo esencial por el apilamiento de coladas de lava de escasa potencia emitidas desde dichos centros, sobre las que destacan algunos aparatos eruptivos dispersos.

\section{El conjunto volcánico de Montaña Cascajo}

La erupción se desarrolló en el cruce de varias fracturas, una principal NO-SE y otra secundaria NE-SO, generando un cono en herradura abierto al noroeste a favor de la pendiente, asimétrico, menos alto hacia el norte, y más regular hacia el sur, compuesto por escorias sueltas y soldadas, bombas volcánicas, jirones de lava y lapilli.

Es un conjunto volcánico de dinámica estromboliana, aunque es evidente que tuvo comportamientos finales eminentemente hawaianos con la formación de la charca lávica y los salideros efusivos meridionales. La estructura interna del cono principal muestra una secuencia de capas fruto de las combinación de etapas estromboefusivas y explosivas durante su construcción, que culminaron con fases mixtas en el interior del cráter y efusivas en el flanco sur del volcán.

\subsection{El edificio volcánico: el cono y el cráter}

Montaña Cascajo constituye un cono volcánico abierto, con cráter en herradura (Fig. 2) de unos 105 metros profundidad y un diámetro mayor y menor de 510 y 240 metros respectivamente. En su interior podemos diferenciar, en relación con el tipo de dinámica predominante - explosiva o no- que ha experimentado el mismo dos ámbitos: uno caracterizado por la profusión de embudos explosivos de escorias y que ocupan fundamentalmente los sectores más altos de la boca eruptiva, y otro, localizado a menor altitud, inmediatamente después de éstos, compuestos por derrames lávicos caóticos de varios metros de potencia totalmente tapizados por piroclastos proximales procedentes de las fases explosivas posteriores; a continuación de éstos, existe una charca de lava que desbordó el cráter, estos derrames junto con las lenguas procedentes de los centros efusivos surorientales son los responsables de las emisiones lávicas de este volcán. Este hecho denota una especialización de los focos eruptivos en función de la localización altitudinal en el interior del cráter. Así, con un desnivel de más de 90 metros entre unos centros y

1 Se consideran como subhistóricos los conjuntos volcánicos generados entre los años 500 a.n.e. y el 1500 d.n.e. aproximadamente (Soler y Carracedo; 1986). 
otros, en los superiores predomina la actividad explosiva, mientras que en los inferiores es la efusiva, aspecto que ha sido constatado en otras ocasiones tanto para el volcanismo histórico (Romero; 1991 y 1992) como subhistórico (Dóniz; 2000).

En el interior del cráter se reconocen, al menos, cuatro centros explosivos de caracteres morfológicos similares y proporciones diferentes que constituyen un rosario de focos alojados en el interior del cráter y que se presentan, tres de ellos, de forma yuxtapuesta unos respecto de otros poseyendo paredes comunes que actúan también como umbrales. El cuarto, alineado con los anteriores, se ubica en torno a unos 100 metros pendiente abajo de éstos. Se trata de un embudo explosivo mínimamente insinuado, elongado, con una profundidad y un diámetro mayor de 3 y 20 metros respectivamente y compuesto por escorias soldadas a modo de plastrones superpuestos.

El resto de las bocas explosivas están constituidas por escorias sueltas, plastrones, jirones, bombas de diverso tamaño y morfología y lapilli. En ocasiones, en las paredes septentrionales de los embudos II y III, las escorias parecen estar buzando hacia el interior del cráter, se trata de escorias fluidales que cubren las paredes (Fig. 3) que al estar plásticas en el momento de la emisión fluyen dando la sensación de rebabas. Frente a la homogeneidad de los productos volcánicos que componen estos cráteres explosivos, la relativa diversidad en cuanto a las dimensiones, pero sobre todo, en relación a la morfología es lo más característico de los mismos.
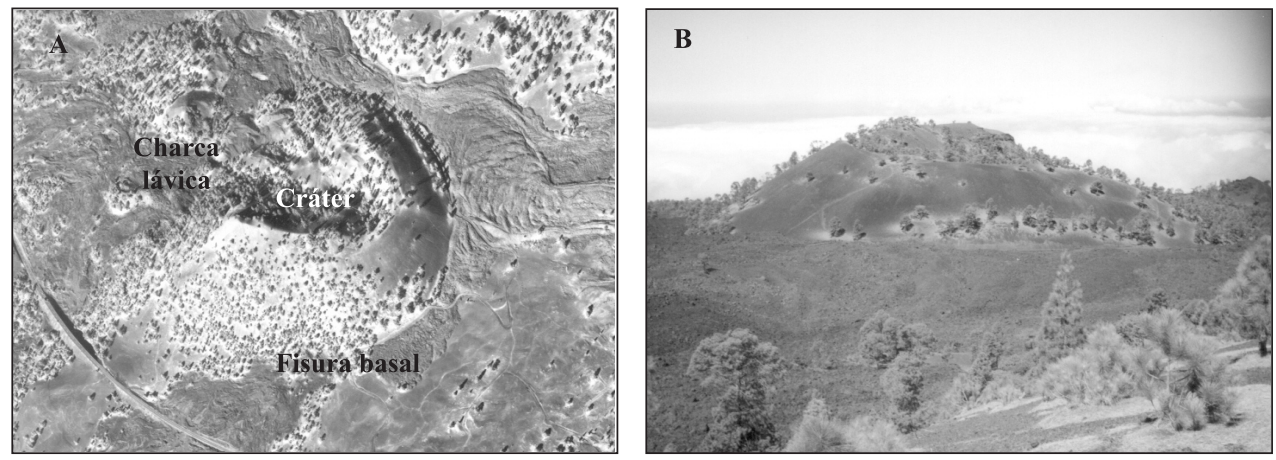

FIguRa 2. Fotografía aérea del conjunto eruptivo de Montaña Cascajo (A) y panorámica del mismo desde el noreste (B)

Los embudos I y II son más o menos simétricos, mientras que el tercero está alargado según la fractura de rumbo NO-SE, la misma que guía la construcción de Montaña Cascajo y la dorsal de Bilma. En cuanto a su envergadura, la boca explosiva número II presenta las mayores dimensiones, éstas están en torno a los 50 metros de profundidad por los $60-70 \mathrm{~m}$ de diámetro, el resto no supera los 20 metros de profundidad ni los 40-50 m de diámetro mayor.

Evidentemente los cuatro embudos explosivos desarrollaron una dinámica eminentemente piroclástica de caracteres estromboexplosivos, ahora bien, no todos ellos con la misma intensidad y frecuencia, ésta debió ser mucho más importante en los cráteres explosivos de mayores proporciones. 
Los embudos II y III debieron de funcionar coetáneamente puesto que, aunque es prácticamente imposible determinar si las escorias y las bombas que tapizan sus fondos corresponden a uno o a otro, si comparten una de las paredes. Con toda probabilidad, el relleno de los cráteres explosivos está en relación, sobre todo, con procesos de dinámica de vertiente y con fenómenos de autoobturación; es más, los piroclastos están graduados según su tamaño que es una de las características básicas de este tipo de fenómenos. El tercer embudo, localizado a mayor altitud, debió ser el primero en iniciar su actividad explosiva, mientras que el cuarto, al estar espacialmente distanciado, es muy difícil establecer la relación cronoestratigráfica con las restantes bocas explosivas.

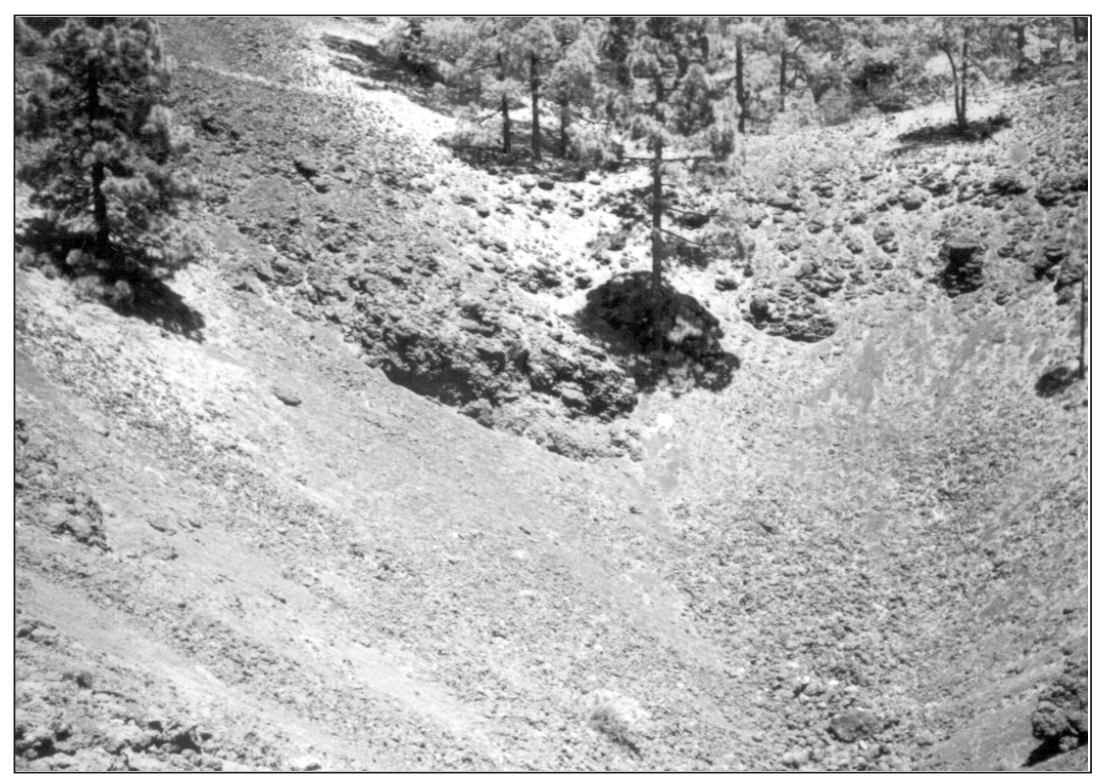

Figura 3. Embudo explosivo III del cráter de Montaña Cascajo

Además, los embudos, las acumulaciones piroclásticas y las cortas lenguas de lava escoriáceas derramadas al norte, que desdibujan la continuidad espacial de las paredes cratéricas de Cascajo, le otorgan a la boca eruptiva principal un aspecto caótico al que contribuye, más aún si cabe, la presencia de numerosos paquetes escoriáceos muy cementados desplomados de las paredes interiores y de la cima de la boca principal.

\subsection{Las coladas de lava}

Las emisiones de lava se producen tanto desde el cráter principal, como de la fisura efusiva suroriental. Desde la boca principal se emiten grandes volúmenes lávicos caóticos, de morfologías escoriáceas, totalmente cubiertos por piroclastos y que se dirigieron hacia el norte y oeste. En el primer caso, las cortas pero potentes lavas en bloques desbordan la pared cratérica y el flanco septentrional del cono desdibujándolo e individualizándolo en dos tramos de dimensiones variables, pero morfológicamente muy similares. Por el contrario, las corrientes que se derramaron hacia el oeste son las que generan la «charca o mar de lava». 
La charca de lava es la viva expresión de un mar en ebullición petrificado donde la presencia de costras pahoehoe asociadas a grietas de resalida que aparecen rotas, inclinadas y giradas, los tubos y jameos, las morfologías de transición y a con diversos niveles de calado, los canales y muros de enfriamiento, junto con las grietas de retracción de varios metros de profundidad y longitud, indicativas de que la lava debió de estar represada, son causantes de la enorme variedad de formas de detalle de esta superficie lávica que se asemeja a un océano encabritado. Hacia el norte y en la base del tramo del edificio piroclástico que quedó individualizado y que no fue sepultado por las lavas del interior del cráter principal, se emite, a través de una grieta de resalida, una colada desgasificada en bloques irregulares y de aristas vivas de unos 60 metros de largo por unos $30 \mathrm{~m}$ de ancho.

Este mar se desborda hacia poniente a lo largo de todo su perímetro generando una cascada de lavas que contribuye a diversificar la morfología de estas corrientes y donde los caracteres más o menos pahoehoe del interior y de las proximidades del desbordamiento, dejan paso a los rasgos superficiales transitorios con canales perfectamente visibles cerca de la cascada y éstos, a los paisajes efusivos de caracteres aa (Fig. 4) que alcanzan varios kilómetros al oeste del conjunto eruptivo abrazando, en su recorrido, a otros edificios eruptivos como los volcanes de Guirres o Abejera (Fig. 5).

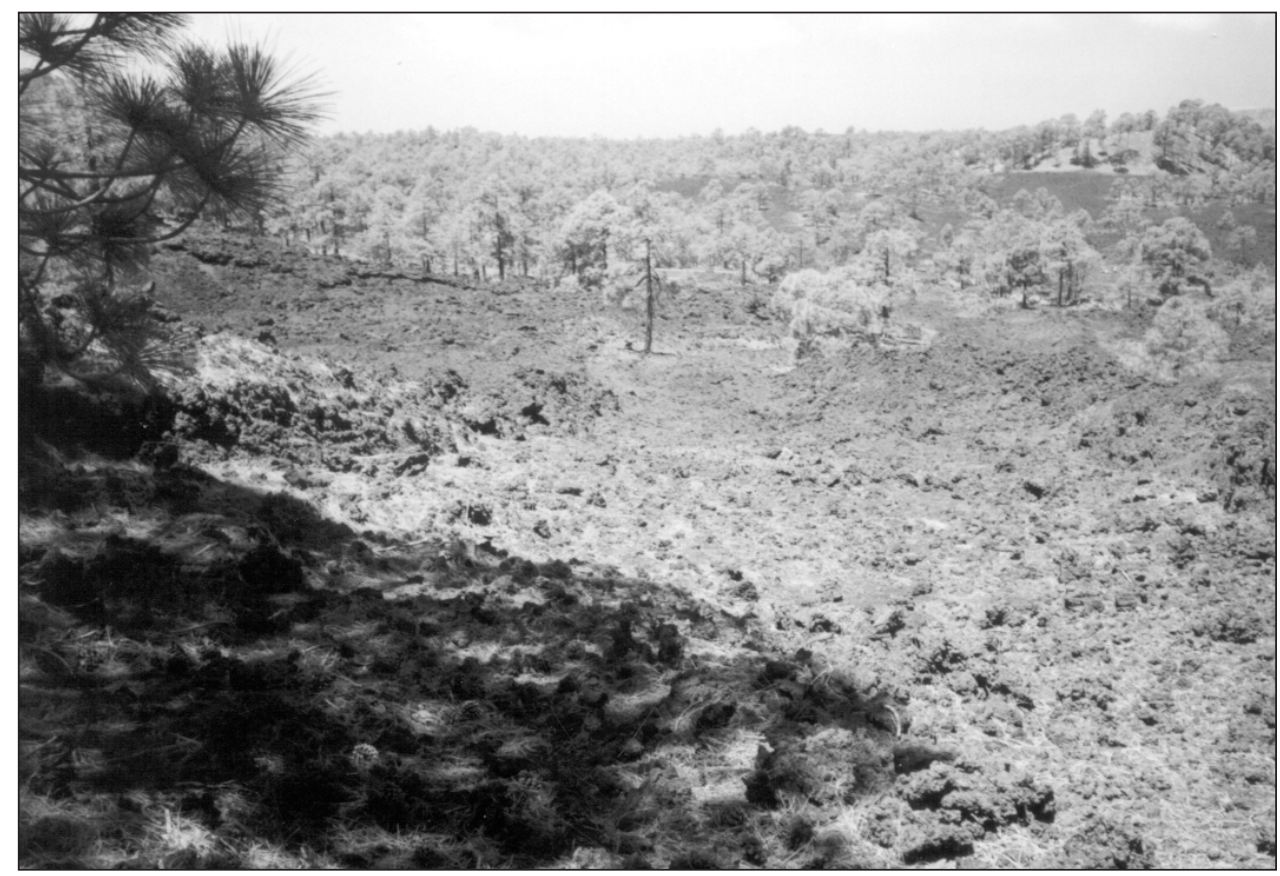

Figura 4. Canal lávico desdibujado en las lavas del volcán Cascajo que funcionó como vía de evacuación de la charca de lava cuando se desbordó

La presencia de varios canales de lava reutilizados en la zona de la cascada, es indicativo de que fueron vías de evacuación en los distintos momentos en los que la charca se desbordó. Algunos desbordamientos tuvieron mínimas repercusiones espaciales, pues existen canales en los que el encauzamiento de las lavas es tan insignificante que no llegaron a 


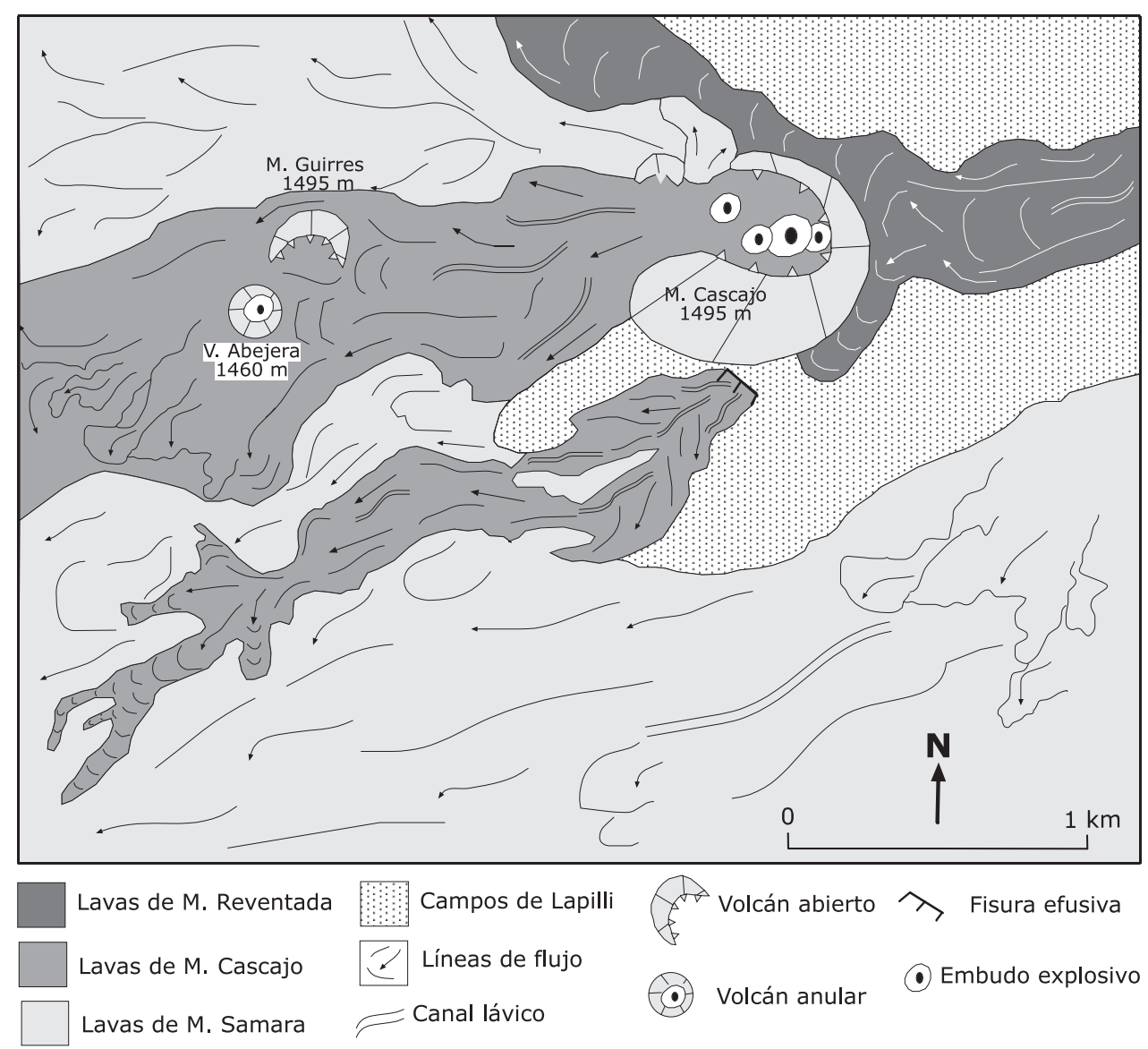

FIgURA 5. Croquis morfológico del edificio volcánico y la fisura efusiva de Montaña Cascajo

recorrer la totalidad de la longitud del mismo y mucho menos rellenarlo. El progresivo desdibujo de los muros laterales de enfriamiento de los canales pendiente abajo son responsables de la apertura y digitación de las lavas pues actúan a modo de torrenteras, de manera que allí donde los muros laterales de enfriamiento desaparecen las lavas se abren adoptando formas pseudotriangulares.

Adosada al flanco SSE del cono principal y alineada según la directriz de rumbo NOSE existe una pequeña fisura efusiva de unos 25-30 metros de largo y al sur de la misma, un rosario de pequeños focos eruptivos. Tanto la grieta como los hornitos desarrollaron comportamientos eruptivos de rasgos hawaianos especializados en la emisión de lavas. Sin embargo, la presencia de las paredes escoriáceas que caracterizan, sobre todo a los hornitos, son indicativos de una reducida actividad explosiva que, a modo de proyecciones intermitentes en la fisura, aunque más intensa y mucho más frecuentes en los hornitos, se limitaba a la expulsión de paquetes de lava fundida con proyecciones de tan sólo varios 
metros de altura. Estos flancos son de reducidas dimensiones, están constituidos por la acumulación y superposición de lavas, jirones, plastrones y escorias y disponen de formas asimétricas como resultado del constante derrame de las lavas hacia el sur que propicia que la pared escoriácea sólo esté presente en el sector opuesto a la salida de las mismas. La menor envergadura de las paredes de los edificios de la fisura respecto a la de los hornitos está en relación con que la actividad explosiva de éstos fue más importante que la de la grieta efusiva.

Las otras acumulaciones de piroclastos en este sector se localizan al SE del rosario de hornitos; se trata de un montículo de lapilli con algunas escorias cuya génesis puede estar relacionada, bien con la presencia de una superficie lávica previa (probablemente de $\mathrm{M}$. Samara) que con posterioridad fue sepultada por los piroclastos de Cascajo quedando en resalte y no pudiendo ser cubierta ni por las lavas emitidas desde la fisura ni las procedentes de los hornitos; o con un túmulo lávico asociado a los primeros derrames procedentes desde la fisura y posteriormente tapizados por los materiales de proyección aérea procedentes de los embudos explosivos del interior del cráter principal.

Morfológicamente la fisura efusiva es mucho más simple que el conjunto de hornitos de resalida. Estos últimos dibujan una trayectoria semicircular en la que sobresalen cuatro pequeños arcos escoriáceos de dimensiones variables, pero reducidas, que se corresponden con otras tantas bocas efusivas que, en algunos puntos de la grieta, alcanzan los 2 ó 3 metros de profundidad. Frente a la relativa continuidad morfoespacial de los localizados hacia el norte, el ubicado al NO está asociado a uno de los muros laterales de las lavas pahoehoe emitidas desde la fisura efusiva y edificado sobre un tubo volcánico. En este caso, y a diferencia del resto de los hornitos, al emplazarse en un sector culminante la emisión de las lavas a favor de la máxima pendiente se realiza en dos direcciones; hacia el sureste vierte la mayor parte de las cortas lenguas de lava y, al noroeste, a través de un canal lávico de un metro de ancho, se emiten pequeñas lenguas.

Actualmente la grieta efusiva está desplomada y debido a como se disponen y adaptan los flujos pahoehoe en las paredes internas de la depresión de la fisura, propio de materiales incandescentes y plásticos, se deduce con toda probabilidad que el hundimiento de la misma tuvo que desarrollarse durante el propio paroxismo, es más, incluso se podría aventurar que existen relaciones genéticas entre el vacío y súbito desplome de la grieta efusiva y los hornitos de resalida.

Estas construcciones surorientales emiten coladas delgadas, de gran longitud y extensión superficial, de rasgos pahoehoe con morfologías superficiales en losas, en tripas y cordadas y sobre las que se reconocen numerosos túmulos, tubos y jameos volcánicos. Todos estos caracteres, el modo en como se disponen, se articulan y se organizan, es propio de terrazas lávicas como las localizadas en el flanco meridional del estratovolcán de Pico Viejo. A medida que las lenguas de lava se alejan de los puntos efusivos adquieren morfologías superficiales de tipo $a a$. En los tramos donde la pendiente comienza a acentuarse, que coincide con los sectores donde se produce la transición de las lavas pahoehoe hacia $a a$, encontramos formas de detalle muy curiosas entre las que destacan las reducidas cascadas de lava que en el talud conectan con canales lávicos que encauzan los fluidos y que luego desaparecen provocando que las lavas se expandan y los pequeños tubos volcánicos con doble galería y jameo que pueden alcanzar los 2 ó 3 metros de profundidad y que se estrechan a medida que ésta aumenta.

Por último, señalar que en este sector del volcán existen recubrimientos parciales por productos de proyección aérea que enmascaran la topografía inicial. Este hecho hace pensar, dada la juventud visible de este sector del conjunto eruptivo, junto con la charca de lava del cráter en herradura, que el paroxismo de Montaña Cascajo debió finalizar con 


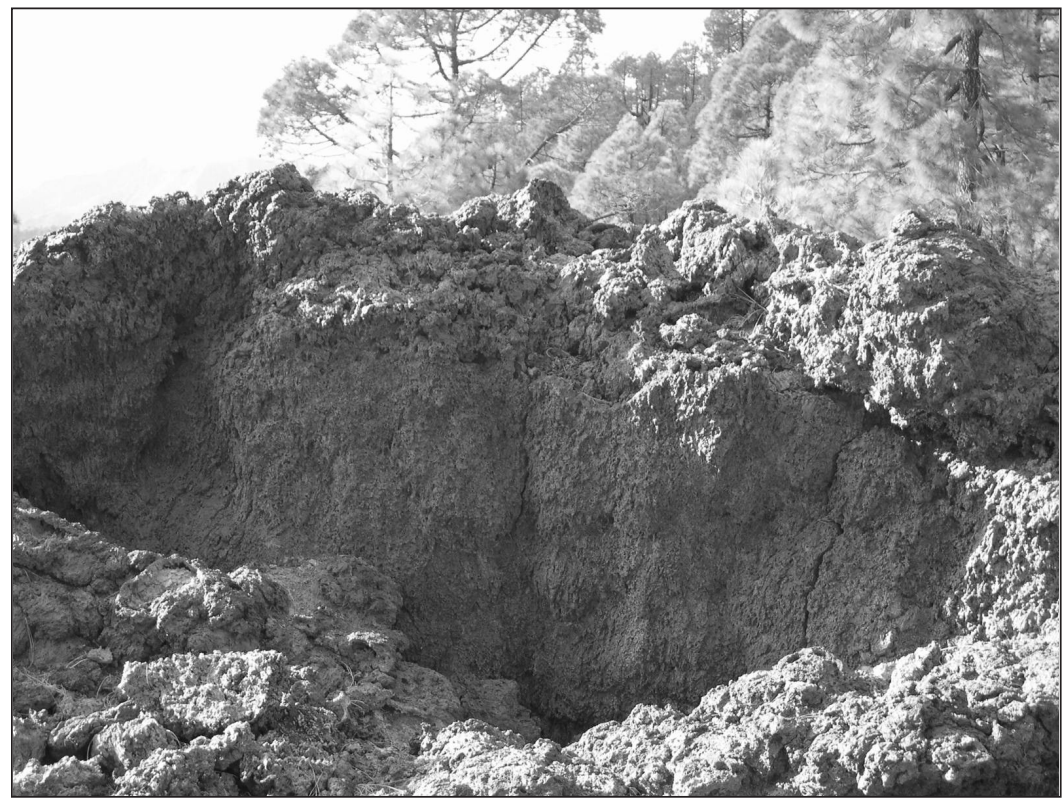

FIGURA 6. Boca eruptiva del hornito nororiental

etapas explosivas asociadas a los embudos del interior del cráter y que también son los causantes de los piroclastos que tapizan las lavas del interior de la boca principal.

\subsection{Los depósitos piroclásticos}

Los materiales de proyección aérea de la erupción de Cascajo debieron cubrir superficies importantes, sin embargo, establecer su área de dispersión es muy difícil debido a que los diferentes campos de piroclastos existentes en los alrededores más próximos del conjunto eruptivo pueden corresponder a cualquiera de los demás paroxismos recientes de este tramo del eje de la dorsal de Bilma-Abeque. Ahora bien, la presencia del cráter junto con los numerosos embudos explosivos instalados en él, son indicativos de que la actividad explosiva existió y, por lo tanto, son responsables de las emisiones de piroclastos que se acumulan al noreste, este, sureste y sur del edificio eruptivo. En todos, excepto el sector meridional, la presencia de bombas de todo tipo y tamaño y las escorias es una característica común.

Los materiales de proyección aérea de Montaña Cascajo cubrieron parte de los derrames lávicos de volcán subhistórico de Samara ubicado unos 200 metros por encima de éste y en torno a unos dos kilómetros de distancia del mismo. En los sectores nororientales, orientales y surorientales, el espesor de los lapilli es considerable, en torno a 1-1,5 m, y cubren prácticamente la colada excepto en algunos puntos concretos en los que se pueden observar las protuberancias escoriáceas de las lavas de Samara. Hacia el Sur de Cascajo, estos productos se concentran en el sector interlávico generado por los derrames procedentes del cráter principal y la fisura efusiva del volcán Cascajo, aquí, la potencia es mayor pero el desarrollo espacial es mucho menor, de manera que dan lugar a un espigón de lapilli que, a medida que se aleja de los centros explosivos, es menos importante. 


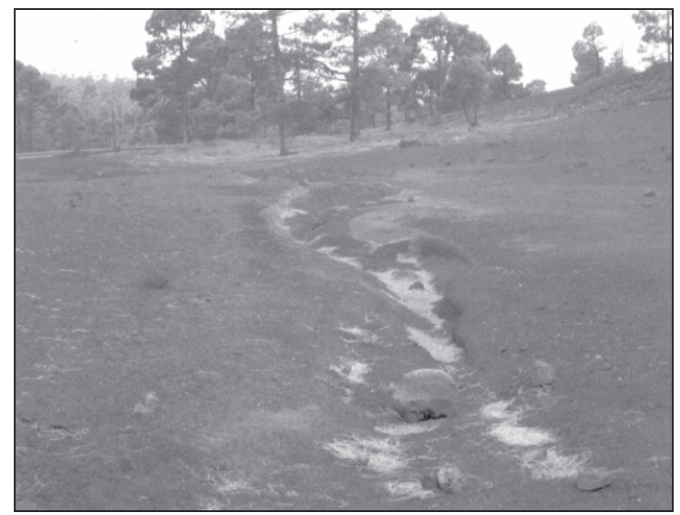

FiguRA 7. Campos de lapilli con huellas de erosión torrencial

\section{Evolución geomorfológica}

Por lo común, las erupciones basálticas que dan lugar a edificios volcánicos simples como el de Montaña Cascajo se inician con fases explosivas que, a medida que transcurre el tiempo, funcionan de manera coetánea, o no, con las etapas efusivas, para finalizar con pulsos explosivos. En este caso, sin embargo, la presencia de paquetes piroclásticos fundamentalmente de escorias estratificados, muy homogéneos y compactos puede asociarse con una fuente de lava inicial que pudo alcanzar varias decenas de metros de altura, muy similares a las descritas para algunos volcanes históricos como los de Fasnia y Chinyero en Tenerife, o San Juan en La Palma (Romero; 1991). Con posterioridad, la columna cae y se incorpora a las corrientes lávicas.

$\mathrm{La}$ apertura de la pista forestal en el flanco NE del cono nos permite observar, al corte, la estratificación de los piroclastos en los dorsos del edificio. Se identifican, al menos, tres niveles distintos relacionados con otros tantos pulsos explosivos en los que el tamaño de los materiales de proyección aérea disminuye desde la base hacia el techo, dando paso a una capa edafizada que se corresponde con los estadios iniciales de la formación de un suelo y cubierta por bloques escoriáceos heterométricos generados por los procesos de dinámica de vertiente (Fig. 8).

La evolución geomorfológica del conjunto volcánico viene caracterizada por un predominio casi absoluto de la morfogénesis mecánica en relación con su edad, ubicación en el piso montano seco y escaso recubrimiento vegetal. Las remodelaciones debidas a los procesos de gravedad, los efectos del viento, las incisiones torrenciales, el periglaciarismo, junto con la acción del hombre, son protagonistas de las modificaciones fisonómicas más contundentes sobre el edificio volcánico y sus derrames lávicos. En los flancos nororientales del cono, sin embargo, se observan superficies de alteración indicativas de un proceso edafogenético inicial.

Las formas debido a la dinámica de vertiente se circunscriben casi exclusivamente a las paredes internas del cráter. La caída de los paquetes de escorias masivas sumitales propicia que hacia la base de las paredes de la boca eruptiva se acumulen, mientras que en los tramos medio-altos de las mismas los productos predominantes sean los lapilli. En la pared cratérica meridional se han descolgado paquetes escoriáceos estratificados que alcanzan dimensiones considerables de más de dos metros de altura por más de tres de largo, en este 


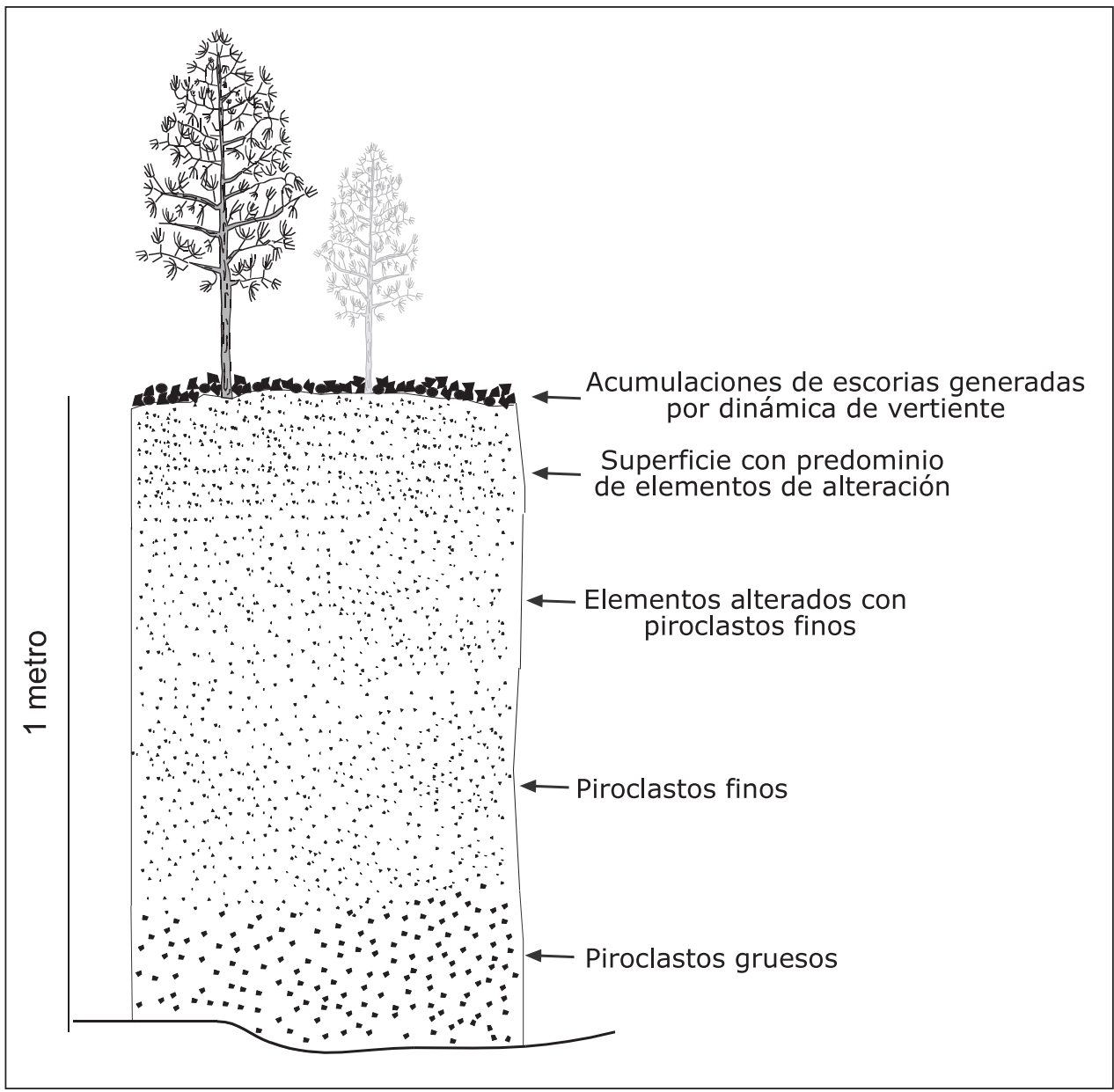

FiguRa 8. Perfil del flanco noreste del volcán de Montaña Cascajo sin indicación de la pendiente, que en este dorso está en torno a los $30^{\circ}$

caso, la caída está desencadenada, entre otros procesos, por el propio fenómeno explosivo que las desestabiliza y caen.

En los flancos del cono y asociado al escaso recubrimiento vegetal y la fuerte pendiente de los mismos y desencadenado por los procesos de gravedad y la escorrentía, existen importantes acumulaciones de escorias removilizadas de diferente tamaño que alternan con finos que se acumulan en la base de los dorsos.

Montaña Cascajo se inserta en la unidad morfohidrológica de las Cumbres de AbequeLas Cañadas donde en torno al $80 \%$ de su superficie se corresponde con áreas sin drenaje o con él incipiente (Romero et al 1999), ello es consecuencia del elevado número de conos volcánicos por $\mathrm{km}^{2}$, existiendo un predominio casi absoluto de las formas volcánicas directas. En el edificio volcánico, dado su juventud y localización, la erosión hídrica desempeña un papel secundario en las transformaciones posteruptivas. En el aparato volcá- 
nico se reconocen sólo tres pequeñas incisiones de unos $0,3 \mathrm{~km}$ de longitud media, dos de ellas localizadas en el cráter y la tercera en el dorso meridional, con un orden máximo de uno y una densidad de drenaje e índice de frecuencia de $2,24 \mathrm{~km}$ de barranco $/ \mathrm{km}^{2}$ y 7,4 cauces $/ \mathrm{km}^{2}$ respectivamente. Relacionado tanto con las aguas de escorrentía como con la apertura de la pista forestal, en los campos de lapilli encontramos varios flujos de derrubios de escasas dimensiones pero con un gran significado morfogenético.

Las acciones eólicas se manifiestan tanto en el aparato eruptivo como en los campos de lapilli y las formas más características son los ripples y los reg. En los afloramientos de escorias masivas del borde del cráter se observan huellas morfológicas actuales de la escasa actividad periglaciar, limitada a los procesos de gelifracción que provocan la fragmentación de las escorias aprovechando los numerosos niveles de debilidad de las mismas.

Como consecuencia de todo ello, la actuación de la morfogénesis y la edafogénesis en este territorio se traduce en toda una gama de procesos y formas erosivas que sólo introducen retoques puntuales en la morfología actual de la construcción eruptiva, por tanto, son las formas volcánicas directas las que caracterizan los rasgos geomorfológicos de Montaña Cascajo.

\section{Discusión}

El conjunto de Montaña Cascajo no es, sin duda, el más reciente de la dorsal de Bilma puesto que en ella han tenido lugar numerosas erupciones subhistóricas e históricas posteriores, sin embargo, su importancia deriva, de un lado, de la originalidad geomorfológica y de la riqueza de formas volcánicas directas reconocibles y, de otro, en que después de unos 1700 años tras el cese de la actividad volcánica que lo edificó, éste presenta, aún cuando lo que predomina es la morfología original, huellas evidentes de su deterioro por los diversos procesos de erosión, que lo convierten en un laboratorio natural para estudiar la evolución de su paisaje.

Ahora bien, independientemente de cuales son los factores que regulan la dinámica morfogenética de los edificios volcánicos simples todos los autores coinciden en individualizar dos etapas en las fases de modelado (Romero, 1991; Inbar et al, 1994; Beltrán, 2000). La primera de ellas se produce inmediatamente después de finalizar la erupción y durante la misma tienen lugar importantes y rápidas remodelaciones de la forma del volcán asociadas con el enfriamiento y asentamiento de los materiales eruptivos. En la segunda etapa, el desmantelamiento de los conjuntos volcánicos es mucho más lento, pero los cambios son más importantes y de mayor desarrollo como consecuencia de la acción prolongada de los agentes externos.

Como es lógico, Montaña Cascajo se halla inmersa en esta segunda fase, ahora bien, las transformaciones más contundentes de su paisaje dependen del tipo de proceso dominante (erosión torrencial, eólica, dinámica de vertiente, periglaciarismo, etc.) y de dónde y cómo actúa éste (sobre el cono piroclástico, sobre las superficies lávicas o sobre los campos de lapilli). En este sentido, la desigualdad más llamativa del aspecto actual de cada uno de los ámbitos descritos del conjunto volcánico está en relación con el mayor o menor protagonismo de la dinámica morfogenética que se combina con la colonización vegetal. Estas diferencias no se debe a que ambos procesos sean siempre excluyentes, pues como veremos a continuación, las formas de modelado están presentes con diferentes grados de evidencia tanto en el cono y en los campos de piroclastos como en las superficies lávicas.

En el edificio volcánico y en el cráter las mayores repercusiones fisonómicas se deben a los procesos de dinámica de vertiente y al desmantelamiento torrencial y, en menor medida, a la erosión eólica, la edafogénesis y el periglaciarismo, este último con una 
localización muy puntual en aquellas escorias masivas sumitales que sobresalen. Algo similar sucede en los campos de piroclastos, con la peculiaridad de que en éstos las transformaciones debidas a los procesos de gravedad son mínimas y dejan paso a las eólicas que adquieren mucho mayor protagonismo y son las responsables de las removilizaciones de los piroclastos y la formación de superficies de ripples y los pequeños regs. A diferencia del cono y de la boca eruptiva y de los mantos de piroclastos, en las coladas de lava las huellas morfológicas de la erosión son prácticamente imperceptibles y sólo de manera puntual se reconocen los efectos de la torrencialidad, la de los fenómenos de gravead en los muros laterales de los canales lávicos y las acumulaciones de finos en las interdigitaciones de las lavas pahoehoe asociadas al soplo del viento. Todo esto denota que no todos los materiales volcánicos son igual de vulnerables ante los procesos de erosión, a la vez que parece existir una mayor intensidad de un tipo u otro de desmantelamiento en función del sustrato volcánico.

\section{Conclusiones}

El conjunto eruptivo de Cascajo está compuesto por un edificio volcánico y sus correspondientes derrames lávicos y campos de piroclastos. Es un cono monogénico de naturaleza basáltica, constituido por piroclastos y con una altura y un diámetro basal de 150 y 780 metros, respectivamente; éste posee un cráter en herradura abierto al noroeste de unos $105 \mathrm{~m}$ de profundidad por 510 metros de eje mayor desde el que se emitieron importantes volúmenes de lavas pahoehoe, de transición y aa. También cuenta con una fisura efusiva basal a través de la que se derramaron lenguas lávicas de gran desarrollo longitudinal. El paroxismo desarrolló comportamientos explosivos y efusivos de rasgos estrombolianos.

El estudio detallado del paisaje geomorfológico de este conjunto eruptivo subhistórico constituye un magnifico ejemplo para analizar los estadios iniciales de la evolución morfológica que experimentan aquéllos volcanes recientes de Tenerife que poseen afinidades dinámicas, morfoestructurales y ambientales con el de Montaña Cascajo. En estas primeras fases tanto los procesos como las formas asociadas a la morfogénesis y a la edafogénesis juegan un papel secundario en su morfología, por cuanto lo que predominan son las formas volcánicas directas. Ahora bien, conjuntos volcánicos de edades y rasgos morfoestructurales similares a los de Montaña Cascajo pero bajo condiciones ambientales más húmedas, disponen de grados de transformación mucho más rápidos, intensos y evidentes que provocan la pronta desaparición de las formas estructurales originales. Por el contrario, cuando la edad es la variable que cambia se convierte en la responsable de las diferencias fisonómicas entre unos volcanes y otros como ocurre con los restantes conjuntos subhistóricos de Samara, Boca Cangrejo y Montañas Negras en las proximidades de Montaña Cascajo.

\section{Bibliografía}

BELTRÁN, E. (2000): El paisaje natural de los volcanes históricos de Tenerife. Fundación Canaria Mapfre-guanarteme. Las Palmas de Gran Canaria, 274 pp.

CARRACEDO, J. (1979): Paleomagnetismo e historia volcánica de Tenerife. ACT. Santa Cruz de Tenerife, $82 \mathrm{pp}$.

CAS, R. y WRIGHT, J. (1987): Volcanic Succesions: Modern and ancient. Allen y Unwin. London, $528 \mathrm{pp}$.

DEHN, J. (1995): Model of cinder cone formation. Summary of Master's degree.

DOHRENWED, J., WELLS, S. y TURRIN, B. (1986): «Degradation of Quaternary cinder cones in the Cima volcanic field, Mojave Desert, California», en Geol. Soc. Am. Bull. no 97, pp. 421-427. 
DÓNIZ, J., ARMAS, V. y ROMERO, C. (1999): «Organización espacial de la actividad eruptiva en la dorsal volcánica de Pedro Gil (Tenerife, Islas Canarias)», en Actas del XVI Congreso Nacional de Geografía, vol. I. Málaga. pp. 81-89.

DÓNIZ PÁEZ, J. (2000): «Rasgos morfológicos de los volcanes recientes de Montañas Negras en Santiago del Teide», (Isla de Tenerife) en Polígonos. ${ }^{\circ}$ 10, pp. 155-165.

DÓNIZ PÂEZ, J. (2001a): «La remodelación torrencial de los edificios volcánicos basálticos simples de Tenerife», en Actas del XVI Congreso Nacional de Geografía. Oviedo, pp. 154-158.

DÓNIZ PÁEZ, J. (2001b): «La influencia de la topografía en las características morfológicas y morfométricas de los volcanes basálticos monogénicos de Tenerife (Islas Canarias)», en Cuaternario y Geomorfología. $\mathrm{n}^{\circ} 15$ (3-4), pp. 125-129.

DÓNIZ PÁEZ, J. (2002a): «Aproximación a la degradación de los volcanes basálticos monogénicos de Tenerife a través de las técnicas morfométricas», en Serrano, E., García de Celis, A., Guerra, J., Morales, C. y Ortega, M. (eds) (2002): Estudios recientes (2000-2002) en geomorfología. Patrimonio, montaña, dinámica territorial. SEG. Dpto. Geografía UV. Valladolid, pp. 445-454.

DÓNIZ PÁEZ, J. (2002b): «El volcanismo basáltico de la dorsal de Pedro Gil en la isla de Tenerife», en Papeles de Geografía. no 35, pp. 101-114.

DÓNIZ PÁEZ, J. (2004): Caracterización geomorfológica del volcanismo basáltico monogénico de la isla de Tenerife. Tesis Doctoral. Departamento de Geografía. Universidad de La Laguna. 396 pp.

HASENAKA, T. y CARMICHAEL, I. (1985): «The cinder cones of Michoacán-Guanajato, central Mexico: ther age, volume, distribution and magma discharge rate», en Journal of Volcanology and Geothermal Research, $\mathrm{n}^{\circ}$ 25, pp. 104-124.

HOOPER, D. y SHERIDAN, M. (1998): «Computer-simulation models of scoria cone degradation», en Journal of Volcanology and Geothermal Research, $\mathrm{n}^{\circ}$ 83, pp. 241-267.

INBAR, M., LUGO-HUBT, J. y VILLERS, L. (1994): «The geomorphological evolution of the Paricutin cone and lava flows, México, 1943-1990», en Geomorphology, n 9, pp. 57-76.

INBAR, M. y RISSO, C. (2001): «A morphological and morphometric análisis of high cinder cone volcanic fiel -Payun Matru, south-central Andes- Argentina», en Zeitschrift fur Geomorphologie, $\mathrm{n}^{\circ} 45$ (3), pp. 321-344.

MARTÍNEZ DE PISÓN, E. y HIGES ROLANDO, V. (1972): «La escala del tiempo en la evolución geomorfológica del aparato eruptivo del Teneguía», en Estudios Geográficos, n XXX-127, pp. 363-378.

ROMERO, C. (1991): Las manifestaciones volcánicas históricas del Archipiélago Canario. Consejería de Política Territorial. Gobierno Autónomo de Canarias. 2 tomos. Santa Cruz de Tenerife, $1463 \mathrm{pp}$.

ROMERO, C. (1992): Estudio geomorfológico de los volcanes históricos de Tenerife. ACT. Santa Cruz de Tenerife, $265 \mathrm{pp}$.

ROMERO, C.; YANES, A. BELTRÁN, E. y DÍAZ, M.C. (1999): «La incidencia de los factores morfoestructurales en la configuración del sistema de drenaje de Tenerife», Actas del XVI Congreso Nacional de Geografía, vol. I. Málaga. pp. 263-271.

SETTLE, M. (1979): «The structure and emplacement of cinder cone fields», en Am. J. Sci. no 279 , pp. 1089-1107.

SOLER, V. y CARRACEDO, J. (1986): «Aplicación de técnicas paleomagnéticas de corto período a la datación del volcanismo subhistórico de la Isla de Tenerife», en Geogaceta, no 1, pp. 33-35.

THOURET, J. (1999): «Volcanic geomorphology-an overview», en Earth Science Reviews, $\mathrm{n}^{\circ}$ 47, pp. 95-131.

TIBALDI, A. (1995): «Morphology of pyroclastic cones and tectonics», en Journal of Geophysical Research, $\mathrm{n}^{\circ} 100$ (B12), pp. 24521-24535.

WOOD, C. (1980a): «Morphometric evolution of cinder cones», en Journal of Volcanology and Geothermal Research, $\mathrm{n}^{\circ}$ 7, pp. 387-413.

WOOD, C. (1980b): «Morphometric analysis of cinder cone degradation», en Journal of Volcanology and Geothermal Research, $\mathrm{n}^{\circ}$ 8, pp. 137-160. 